\title{
A Thin-Plate Spline Calibration Model for Fingerprint Sensor Interoperability
}

\author{
Arun Ross, Member, IEEE, and Rohan Nadgir
}

\begin{abstract}
Biometric sensor interoperability refers to the ability of a system to compensate for the variability introduced in the biometric data of an individual due to the deployment of different sensors. Poor intersensor performance has been reported in different biometric domains including fingerprint, face, iris, and speech. In the context of fingerprint technology, variations are observed in the acquired images of a fingerprint due to differences in sensor resolution, scanning area, sensing technology, etc., which subsequently impact the feature set extracted from these images. The inability of a fingerprint matcher to compensate for these variations introduced by different sensors results in inferior intersensor matching performance. In this work, a nonlinear calibration scheme based on the ThinPlate Spline (TPS) model is used to register a pair of fingerprint sensors. The proposed calibration technique relies on the evidence of a few image pairs acquired using the two sensors to generate an average deformation model that defines the spatial relationship between the two sensors. This assists in the systematic perturbation of images/features pertaining to one sensor in order to make them compatible with images/features originating from the other sensor. Experimental results using multiple fingerprint data sets confirm the efficacy of the proposed method in addressing intersensor geometric variations.
\end{abstract}

Index Terms-Biometrics, fingerprint, interoperability, calibration, splines, deformation.

\section{INTRODUCTION}

B IOMETRICS refers to the use of distinctive physical (for example, fingerprints, face, iris, hand geometry, and palmprint) or behavioral (for example, gait, signature, and keystroke dynamics) characteristics for automatically recognizing individuals [1]. Among all biometric traits, fingerprints have been extensively used for human recognition and have been studied by both the biometric and the forensic research communities [2]. Fingerprint-based biometric systems are being used in a variety of applications including the US-VISIT ${ }^{1}$ program instituted by the Department of Homeland Security (DHS) and the IAFIS ${ }^{2}$ service developed by the Federal Bureau of Investigation (FBI).

Fingerprints are oriented texture patterns consisting of ridges and valleys present on the surface of an individual's fingertip. The fingerprint impressions of an individual can be imaged by rolling the inked finger surface on paper or by placing the fingertip on the platen of an electronic sensor. Based on the acquisition methodology, the fingerprint impression acquired from a fingertip may be categorized as being a rolled, dab, swipe, or latent print (Fig. 1). Historically, fingerprints have been acquired by smearing ink on the fingertip and creating an inked impression of the finger on paper. The rolled print shown in Fig. 1a is acquired

1. United States Visitor and Immigration Status Indicator Technology.

2. Integrated Automated Fingerprint Identification System.

- A. Ross is with the Lane Department of Computer Science and Electrical Engineering, West Virginia University, Morgantown, WV 26506. E-mail: arun.ross@mail.wou.edu.

- R. Nadgir is with BIO-key International, Inc., 1285 Corporate Center Drive, Suite 175, Eagan, MN 55121. E-mail: rohan.nadgir@gmail.com.

Manuscript received 30 Apr. 2006; revised 25 Feb. 2007; accepted 23 July 2007; published online 3 Oct. 2007.

For information on obtaining reprints of this article, please send e-mail to: tkde@computer.org, and reference IEEECS Log Number TKDESI-0212-0406. Digital Object Identifier no. 10.1109/TKDE.2007.190696. by rolling the finger "nail-to-nail" in order to obtain the "unwrapped" image [1]. Advances in sensor technology now permit the online acquisition of fingerprints using scanners based on optical, capacitive, piezoelectric, thermal, or ultrasonic principles [1], [4]. The sensing area of these scanners can vary from a few square millimeters to a few square inches. The resolution of the acquired image can vary between 250 dpi (for example, Authentec's AF-S2 FingerLoc) and 512 dpi (for example, Digital Persona's U.are.U 4000); scanners that acquire 1,000-dpi images of the fingerprint (for example, Aprilis' HoloSensor) are also available in the market. The fingerprint images acquired using different sensing methods can exhibit significant variations in their geometric dimensions, as well as their photometric composition. This inherent variation in the acquired images is illustrated in Fig. 2, where five different scanners were used to capture impressions of the same fingerprint.

The variations introduced in the acquired images due to differences in resolution, scanning area, sensing technology, etc., impact the features extracted from the images (for example, minutiae points) and, consequently, the templates stored in the database. Most fingerprint matchers are restricted in their ability to compare fingerprints originating from two different sensors resulting in poor intersensor performance [4]. Inferior intersensor performance has been reported not only in the fingerprint domain but also in other domains such as speech, iris, and face. Martin et al. [5] report a significant drop in the matching performance of speech-based biometric systems when the input device is switched from a carbon-button microphone to an electret microphone (and vice versa). The results of the iris recognition test conducted by the International Biometric Group (IBG) [6] suggest that intersensor matching performance is lower than intrasensor performance. In the face domain, the variations introduced due to different cameras are expected to affect the performance of face recognition algorithms as severely as the variations introduced due to 


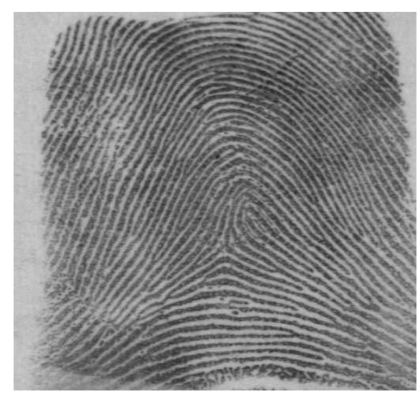

(a)

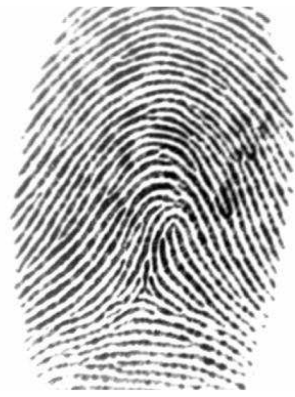

(b)

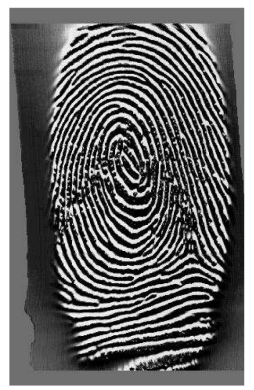

(c)

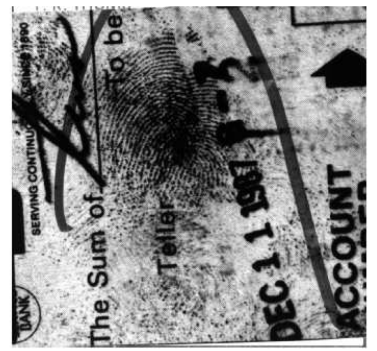

(d)

Fig. 1. Four different schemes for fingerprint acquisition. (a) Rolled print (from the NIST Special Database 4). (b) Dab print (from the FVC 2002 DB1 Database). (c) Swiped sensor print (from the FVC 2004 DB3 Database). (d) Latent print (from [3]).

differing illumination patterns [7]. A study conducted by Faltemier and Bowyer [8] reports degradation in the matching performance of intersensor 3D face recognition. Their study concludes that intersensor 3D face matching may be facilitated under certain limited conditions by compensating for variations in the sampling densities, resolution accuracies, and noise characteristics.

The problem of sensor interoperability, as defined in this paper, cannot be solved by adopting a common biometric data exchange format [9]. Such a format merely aids in the exchange of feature sets between systems/ vendors [10]. However, it does not provide a method to match feature sets obtained from different sensors. Similarly, the Electronic Fingerprint Transmission Specification (EFTS) [11] defines requirements on fingerprint scanner systems and printers that supply fingerprint data to the Integrated Automated Fingerprint Identification System
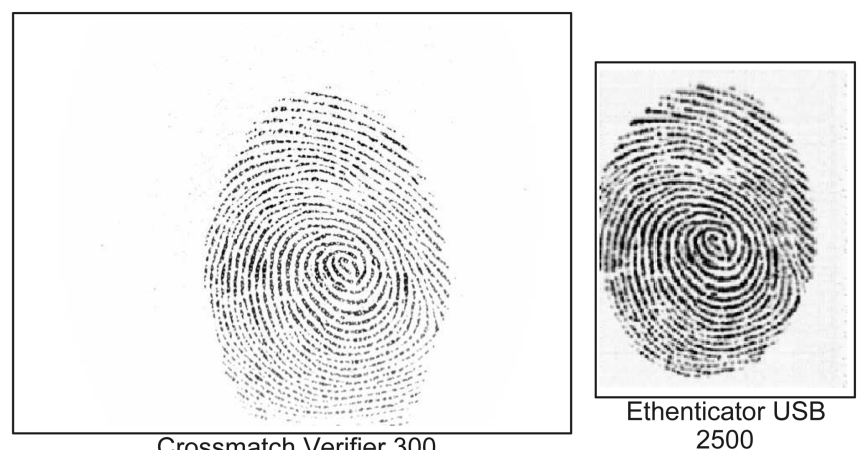

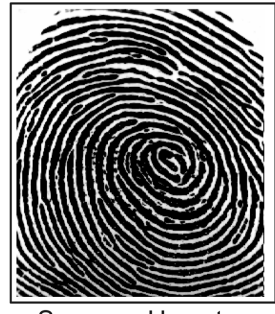

Secugen Hamste

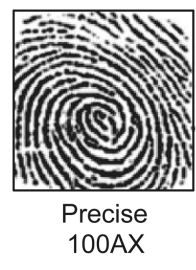

100AX

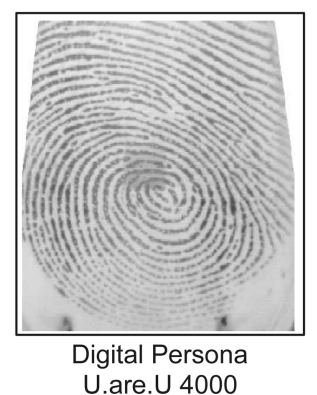

Fig. 2. Visual difference between impressions of the same finger acquired using five different sensors. Crossmatch Verifier 300 (CM), Secugen Hamster III (SG), and Digital Persona U.are.U 4000 are optical sensors; Hamster III is based on a SEIR technology, whereas U.are.U 4000 uses a FTIR technology. Ethenticator USB 2500 is a polymer-based sensor, and Precise $100 \mathrm{AX}(\mathrm{PC})$ is a capacitive sensor.
(IAFIS). The EFTS provides objective criteria for ensuring image quality while maintaining the geometric and spatial integrity of the supplied fingerprint images. However, it does not define a method to compare fingerprint images demonstrating significant differences in geometric and photometric characteristics.

In order to motivate the problem of fingerprint sensor interoperability, we consider the US-VISIT program that obtains fingerprint (and face) information of certain travelers arriving at the nation's airports and seaports. A 500 -dpi optical scanner with a sensing area of $1.2^{\prime \prime} \times 1.2^{\prime \prime}$ is currently being used during the enrollment phase to procure fingerprint images. The introduction of a different sensor during the verification stage might render the current data unusable. The cost of reenrolling individuals every time the sensor is changed will be tremendous. In applications like these, the need for sensor interoperability is paramount and will significantly impact the usability of the system. Thus, the problem addressed in this paper has direct applications in fingerprint-based security systems that employ different types of sensors for fingerprint image acquisition.

Besides changes in sensing technology, the fingerprint acquisition and matching methodology may also vary across systems. Contact-based sensors can obtain rolled, flat, or slap prints of a finger (Fig. 3). The ability to successfully compare rolled prints against, for instance, the associated slap prints is indeed a challenging problem. In this work, we concern ourselves with the interoperability between different sensing technologies (for example, optical versus capacitive) and not between the modes of acquisition (for example, rolled versus flat). In particular, we demonstrate that a simple nonlinear calibration scheme is sufficient to facilitate sensor interoperability. In the proposed framework, the difference between the images acquired using two different sensors is modeled using nonlinear distortions represented using Thin-Plate Splines (TPSs). The use of a nonlinear distortion model that accounts for local warping is appropriate for the following reasons: 1) the physics of the sensing process can introduce distortions unique to each sensor technology; even a versatile matcher may fail to account for such types of distortions, and 2) the nature of the distortion may vary across the sensor due to the arrangement of the sensing elements within the device; thus, a linear global transformation may be unsuitable.

The remainder of the paper is organized as follows: In Section 2, some of the commonly encountered fingerprint 


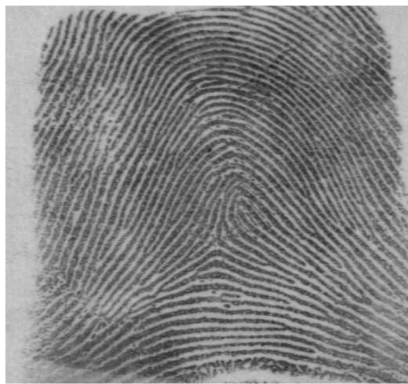

(a)

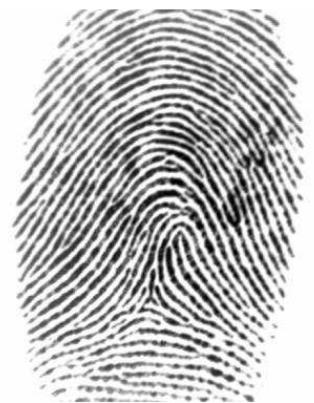

(b)

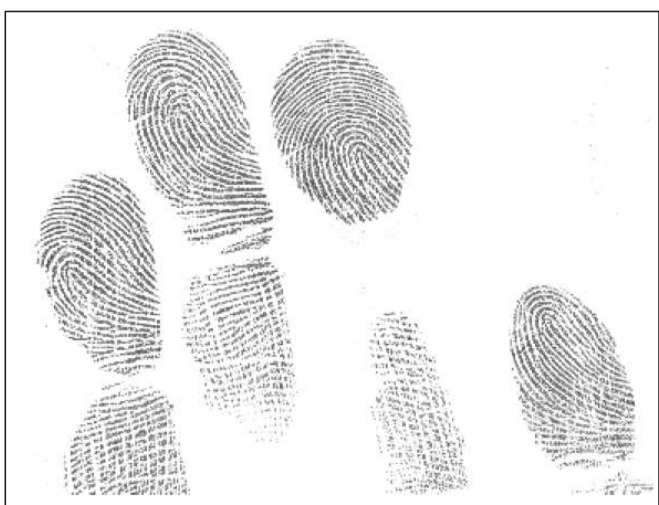

(c)

Fig. 3. Different fingerprint acquisition methods. (a) Rolled print (from the NIST Special Database 4). (b) Flat print (from the FVC 2002 DB1 Database). (c) Slap print [12].

sensing technologies are enumerated. Section 3 discusses two possible approaches to reconcile images originating from multiple sensors. Section 4 presents the average deformation model used in this paper to model intersensor distortions. Section 5 discusses experimental results on the Michigan State University (MSU) and West Virginia University (WVU) database suggesting the efficacy as well as the limitations of the proposed scheme. Section 6 concludes the paper with a discussion on the merits and demerits of our approach.

\section{Fingerprint Sensing Technology}

A variety of fingerprint sensing technologies employing different operational principles are currently available. These include optical (Frustrated Total Internal Reflection (FTIR) and Surface Enhanced Irregular Reflection (SEIR)), capacitive, piezoelectric, temperature differential, ultrasound, touchless, and multispectral sensors (see Table 1). Since optical and capacitive sensors were utilized in this research, a brief description of these two technologies has been included below.

Optical sensors. Optical sensors based on FTIR consist of a glass prism, a light source, and a focusing lens along with a CMOS or a CCD camera. The light is directed onto the surface of the prism, which acts as a platen for the finger to be placed, and the reflected light is focused by the lens onto the camera, which captures the fingerprint image. Light rays are totally reflected from the valleys, giving them a lighter appearance, whereas light rays are not reflected from the ridges, giving them a darker appearance. The focal length of the lens is a deciding factor in the size of the sensor. If a lens having small focal length is selected, optical distortion could be observed in the captured images. Hence, there is a trade-off between the sensor size and optical distortion. Optical sensors are normally bulky because of this setup.

Some optical sensors use a SEIR technique to capture fingerprints. SEIR is based on scattering principles in which light is scattered and reflected from the ridges and not from the valleys. Most of the scattered light is collected, giving the ridges a brighter appearance than the valleys.

Capacitive sensors. These sensors are composed of a number of small capacitive plates placed under the sensor platen. Air acts as the dielectric medium, and the electric field strength is a function of the distance of the fingerprint ridges and valleys from the capacitive plates. The electric field drops off as the inverse of the distance of the plate to the object, that is, the skin [15]. To achieve a certain resolution, a compromise between the size of the capacitive plates and the distance between capacitive plates has to be obtained. Capacitive sensors may suffer from static discharge, which could possibly damage the plates; hence, proper grounding is required.

Each sensing technology introduces its own type of distortions while acquiring the fingerprint image. Thus, deformation in fingerprint images is not only a consequence of the elastic skin interacting with a solid platen/surface during the image acquisition process but also a characteristic of the sensor. Distortions may result in parts of the image being stretched, compressed, or out of focus with respect to the rest of the image. Blurred edges are sometimes observed in images captured using optical scanners. This is due to the relatively larger size of the fingerprint area compared to the first lens in the lens assembly, leading to nonparallel light paths toward the edge of the image [13]. In some acquisition systems, the path lengths of reflected light differ across the length and width of the fingertip. Differences in path lengths can cause part of the image to be wider than the rest of the image-a principle known as Trapezoidal Distortion [13], [16], [17]. In Fig. 2, the image captured using Digital Persona's U.are.U 4000 exhibits this type of distortion. Varying path lengths also generate defocused areas within the captured image. The curvature of the lens assembly can lead to a curved or out-of-focus appearance along the outer edges of the image. Capacitive sensors are prone to noisy artifacts, including noise from the $60-\mathrm{Hz}$ power line and electrical noise from within the sensor. The semiconductor-sensing chips are also sensitive to electrostatic discharge, salt from sweat, and other contaminants, as well as physical wear. Grid artifacts are possible in capacitive sensors. Hence, intrinsic sensor properties introduce distortions in the resulting images.

\section{SENSOR INTEROPERABILITY}

Biometric sensor interoperability refers to the ability of a system to compensate for the variability introduced in the raw biometric data (for example, images) of an individual 
TABLE 1

Fingerprint Sensing Technologies

\begin{tabular}{|c|c|c|c|}
\hline No. & $\begin{array}{l}\text { Sensing } \\
\text { Technology }\end{array}$ & Working Principle & Products \\
\hline 1 & $\begin{array}{l}\text { Optical } \\
\text { (FTIR - } \\
\text { Frustrated } \\
\text { Total Internal } \\
\text { Reflection) }\end{array}$ & $\begin{array}{l}\text { FTIR is based on total internal reflection. } \\
\text { Light is reflected from valleys and not from } \\
\text { ridges. Lens focuses the reflected light } \\
\text { rays onto the camera. Valleys appear } \\
\text { bright while ridges appear dark. [4], [13] }\end{array}$ & $\begin{array}{l}\text { Digital Persona } \\
\text { U.are.U } 4000\end{array}$ \\
\hline 2 & $\begin{array}{l}\text { Optical } \\
\text { (SEIR - Surface } \\
\text { Enhanced } \\
\text { Irregular } \\
\text { Reflection) } \\
\end{array}$ & $\begin{array}{l}\text { SEIR is based on scattering principles. } \\
\text { Light is reflected and scattered from ridges } \\
\text { and not from valleys. Most of the scattered } \\
\text { light is collected, hence ridges appear } \\
\text { bright while valleys appear dark. [13] }\end{array}$ & $\begin{array}{l}\text { Secugen } \\
\text { Hamster III }\end{array}$ \\
\hline 3 & Capacitive & $\begin{array}{l}\text { Air acts as dielectric medium. Capacitance } \\
\text { is a function of the distance of ridges and } \\
\text { valleys from the capacitive plates. [4] }\end{array}$ & $\begin{array}{l}\text { Fujitsu MBF200 } \\
\text { Precise 100AX }\end{array}$ \\
\hline 4 & Piezoelectric & $\begin{array}{l}\text { Current is generated as a function of varying } \\
\text { pressure applied by ridges and valleys } \\
\text { on a dielectric material. [4] }\end{array}$ & $\begin{array}{l}\text { Fidelica } \\
\text { Microsystems } \\
\text { FIS } 3002\end{array}$ \\
\hline 5 & $\begin{array}{l}\text { Temperature } \\
\text { Differential }\end{array}$ & $\begin{array}{l}\text { Current is generated as a function of varying } \\
\text { temperature differentials observed across } \\
\text { ridges and valleys. [4] }\end{array}$ & $\begin{array}{l}\text { Atmel } \\
\text { AT77C101B } \\
\text { Swipe sensor }\end{array}$ \\
\hline 6 & Ultrasound & $\begin{array}{l}\text { Image generated on basis of the response } \\
\text { of the acoustic wave bounced off the } \\
\text { fingertip. No skin contact. [4] }\end{array}$ & $\begin{array}{l}\text { Ultra-Scan } \\
\text { UltraTouch } \\
\text { Model } 203 \\
\end{array}$ \\
\hline \multirow[t]{2}{*}{7} & \multirow[t]{2}{*}{ Touchless } & $\begin{array}{l}\text { 3-D image generated by integrating images } \\
\text { captured by different cameras. }\end{array}$ & $\begin{array}{l}\text { TBS 3-D sensor. } \\
\text { Surround Imaging }(\mathrm{tm}) \\
\text { technology }\end{array}$ \\
\hline & & $\begin{array}{l}\text { Light reflected by ridges is converted into } \\
\text { electrical signals to generate a image. } \\
\text { Contact-less. }\end{array}$ & $\begin{array}{l}\text { TST group } \\
\text { BiRDIIi }\end{array}$ \\
\hline 8 & Multispectral & $\begin{array}{l}\text { Multispectral data is collected under } \\
\text { different illumination angles and polarizing } \\
\text { conditions as well as different wavelengths. } \\
\text { Fingerprint information below the surface } \\
\text { of the skin is captured easily. [14] }\end{array}$ & $\begin{array}{l}\text { Lumidigm } \\
\text { LightPrint } \\
\text { Technology }\end{array}$ \\
\hline
\end{tabular}

due to the deployment of different sensors. In the context of fingerprints, interoperability may be accomplished by two different approaches, as shown in Fig. 4:

1. Distortion compensation model. In this approach, the goal would be to determine and model the physics of the distortion process when a user places her finger on a particular scanner. This distortion would be based on the sensing technology of that scanner, as well as the process employed to convert the sensed data into a raw image. As shown in Fig. 4a, knowledge of the distortion process will permit us to compute the original "undistorted" fingerprint (canonical image). The canonical image may then be used for matching purposes [18].

2. Intersensor distortion model. In the second approach, the relative distortion between images acquired using two different sensors can be computed (Fig. 4b). Modeling the intersensor distortion may be viewed as a calibration problem, and can be accomplished by inheriting the knowledge of corresponding points on the two sensors. This is similar to the camera calibration problem in computer vision wherein the knowledge of corresponding points on a chessboard plane is used for appropriately registering two different cameras, as well as computing the intrinsic parameters for a camera [19], [20], [21], [22]. The intersensor distortion model is similar to camera calibration and is a combination of affine and elastic distortions.

In this work, the second approach indicated above is used to address the problem of interoperability. A TPS model is employed to characterize the intersensor distortion since such a model can account for the affine and the nonlinear aspects of the deformation. The parameters of the intersensor distortion model are estimated based on the evidence of correspondence between the control points 


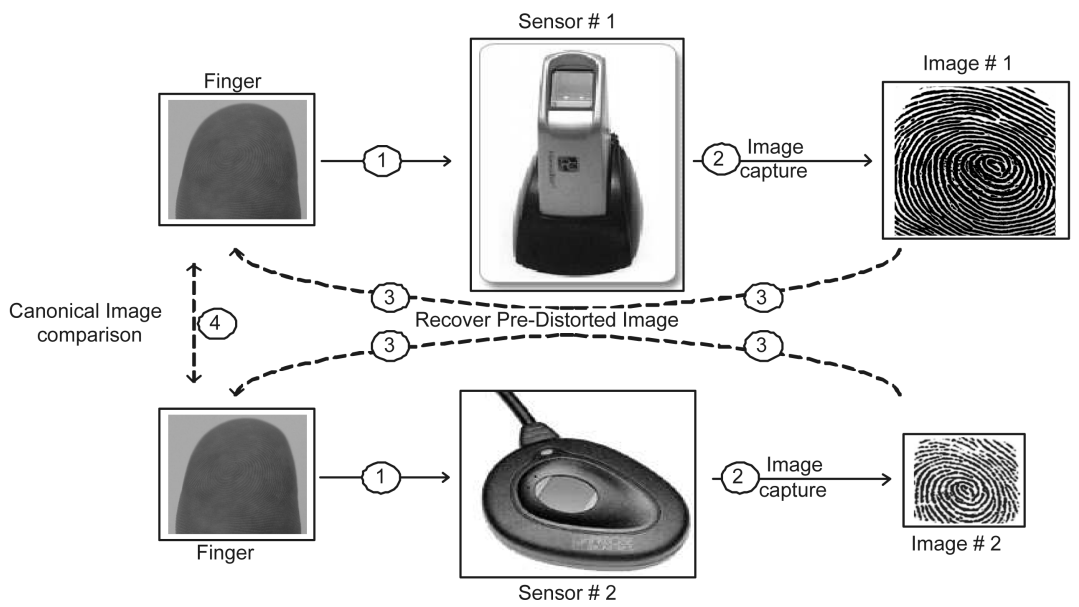

(a)

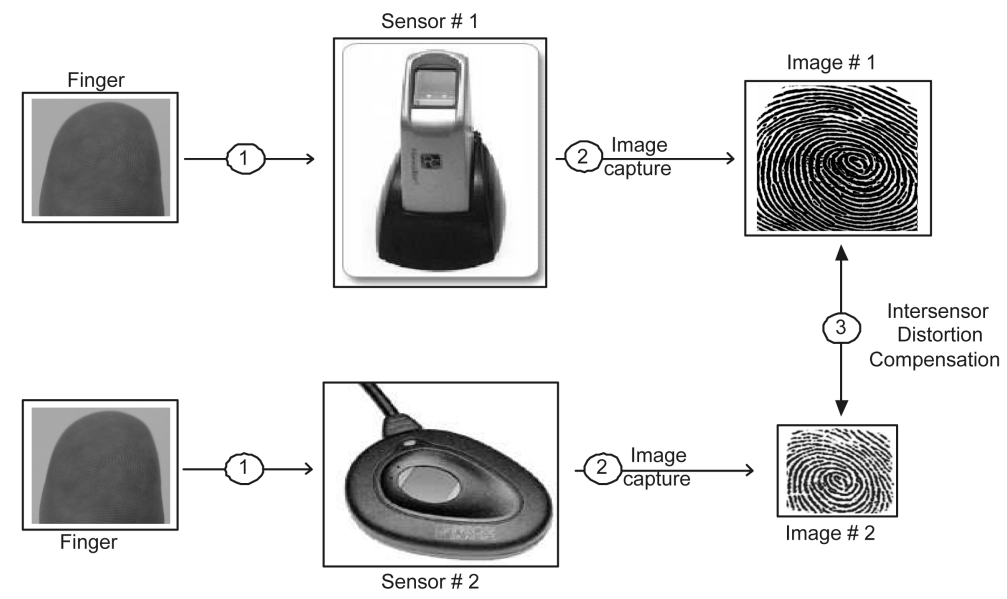

(b)

Fig. 4. Two different ways of facilitating interoperability. (a) Distortion compensation model. (b) Intersensor distortion model. The numbers within the circles denote the sequence of steps.

present on the two sensors. In our approach, corresponding points (control points) are obtained by manually locating minutiae points from a small set of representative fingerprint image pairs (Fig. 5). The control points are selected manually to approximately cover the whole area within the smaller fingerprint image in order to model the distortions occurring in different areas of the representative image. These control points are then used to derive a deformation model that represents the relative distortion

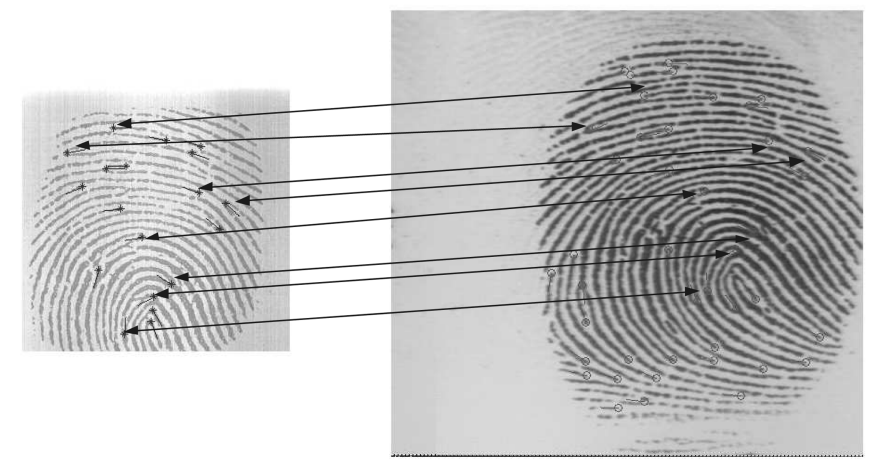

Fig. 5. Minutiae correspondence (manually selected) across representative image pairs serve as inputs to the TPS model. between the two images (Fig. 6). A procrustes analysis of the control points assists in the computation (and subsequent removal) of translation and rotational parameters relating the two representative images. Procrustes analysis is a rigid shape analysis that uses isomorphic scaling, translation, and rotation to find the "best" fit between two shapes defined by some control (landmark) points. In our methodology, a procrustes analysis is used to determine only the translation and rotation parameters. The translation parameters are obtained by aligning the centroids of both sets of control points. If $P=$ $\left\{\left(u_{1}, v_{1}\right),\left(u_{2}, v_{2}\right), \cdots,\left(u_{n}, v_{n}\right)\right\}$ is the set of $n$ control points in sensor 1 and $P^{\prime}=\left\{\left(u_{1}^{\prime}, v_{1}^{\prime}\right),\left(u_{2}^{\prime}, v_{2}^{\prime}\right), \cdots,\left(u_{n}^{\prime}, v_{n}^{\prime}\right)\right\}$ is the corresponding set of $n$ control points in sensor 2, then the centroids are given by $\left(\bar{u}=\sum_{i=1}^{n} \frac{u_{i}}{n}, \bar{v}=\sum_{i=1}^{n} \frac{v_{i}}{n}\right)$ and $\left(\bar{u}^{\prime}=\sum_{i=1}^{n} \frac{u_{i}^{\prime}}{n}, \bar{v}^{\prime}=\sum_{i=1}^{n} \frac{v_{i}}{n}\right)$, respectively. The control points are next zero-centered based on their centroids. The zerocentered $P$ and $P^{\prime}$ are symbolized as $P_{M}$ and $P_{M}^{\prime}$. The optimal rotation between the two point clouds is computed by estimating the orthogonal matrix $Q$ such that the expression $m=\left\|P_{M}-Q P_{M}^{\prime}\right\|$ is minimized. In order to maximize the correlation between the two sets of landmark points, the optimal $Q$ is calculated by the singular value decomposition (SVD) of $P_{M}^{T} P_{M}^{\prime}$, that is, SVD $\left(P_{M}^{T} P_{M}^{\prime}\right) \rightarrow U D V^{T}$, and $Q=V U^{T}$ (where $\mathrm{T}$ is the matrix transpose operator). 


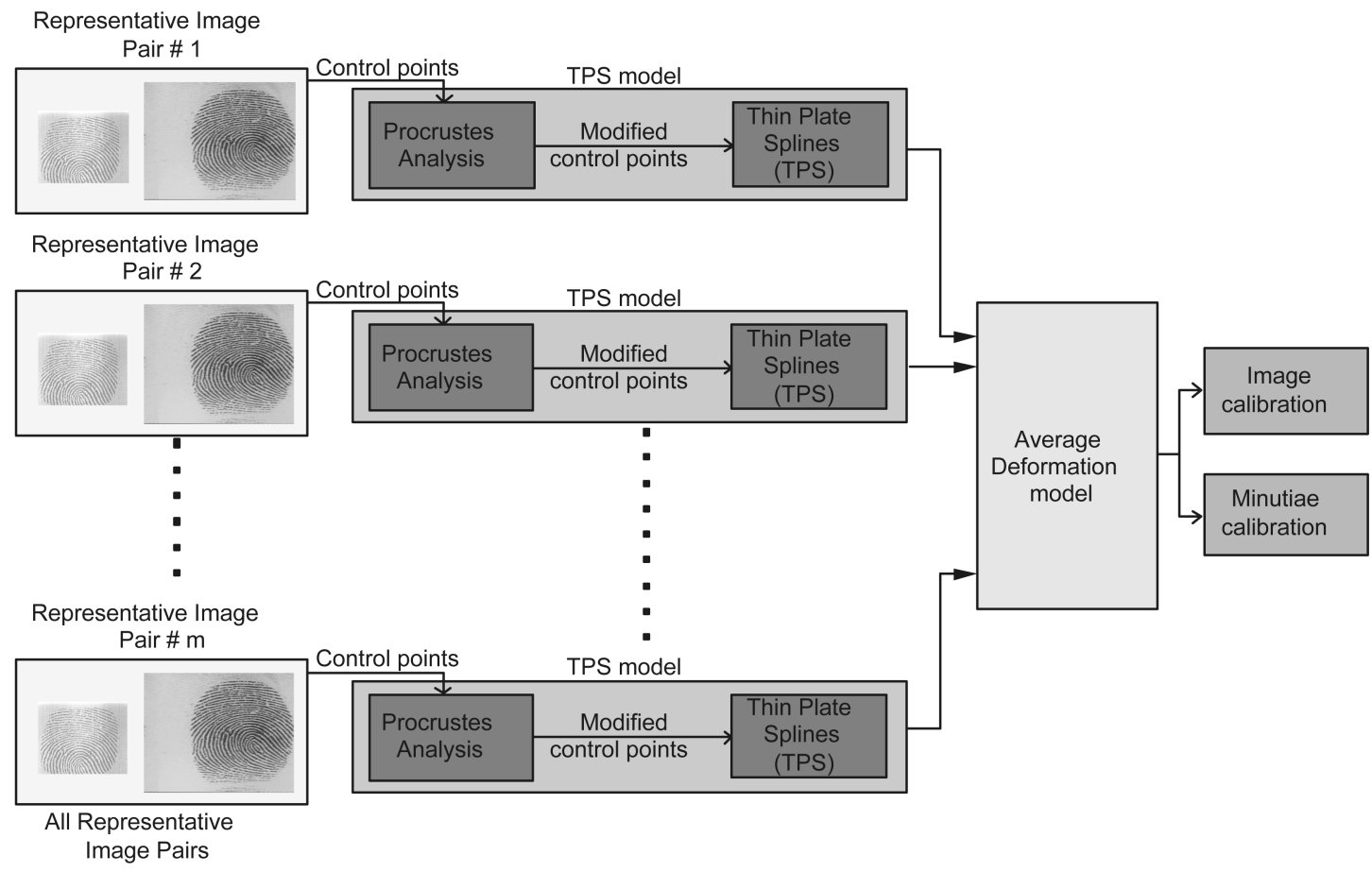

Fig. 6. Manually selected control points from representative image pairs are provided as inputs to the TPS model. The affine and nonlinear parameters derived from the average deformation model are used for image and minutiae calibration during the authentication stage.

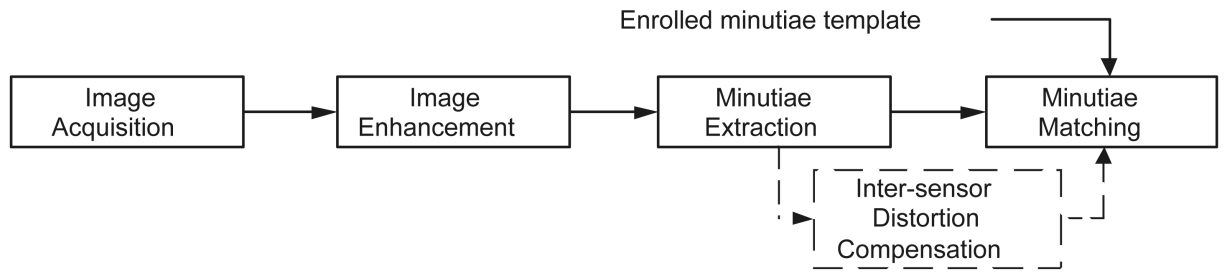

Fig. 7. Minutiae calibration. The intersensor distortion compensation block can be introduced between the minutiae extraction and matching modules to handle intersensor distortions.

The rotation and translation factors are due to variation in finger placement across different acquisitions and do not contribute toward the intersensor distortions. The TPS model, described in the next section, is used to compute the affine and the nonlinear parameters from the translation- and rotation-corrected control points. Having first compensated for the translation and rotation parameters, the TPS deformation parameters can be computed independent of these effects. The TPS parameters are estimated for several representative image pairs based on manually established control points. Multiple sets of TPS parameters aid in generating an average deformation model [23], which defines the perturbation at every pixel (point) on one sensor with respect to the other.

The resulting average deformation model may be used for minutiae, as well as image, calibration. If only the minutiae templates of a sensor are available, then the location and orientation of the minutiae points may be modified. On the other hand, if the fingerprint image itself is available as a template in the database, then the pixel locations of the image can be modified, resulting in a "deformed" image, which is then subjected to the process of feature extraction and matching.

The proposed intersensor distortion model can be incorporated into a minutiae-based matcher for the successful comparison of templates originating from different sensors, as shown in Fig. 7.

\section{Thin-Plate Spline Model}

For a spatial rearrangement of points, the TPS succinctly expresses the dependence of the physical bending energy of a thin metal plate on point constraints [24]. TPS has been used in many 2D [25], [26], [27], [28] and 3D [26], [27], [28], [29] medical imaging applications for appropriate registration. Recently, TPS has been used to model the nonlinear deformations in fingerprints [23], [30], [31], [32], [33], [34].

\subsection{Bending Energy of TPS}

Given a list of corresponding points, the TPS model interpolates the corresponding grid points while maintaining smoothness as defined by the bending energy of the thin metal plate. The smoothness is maintained by minimizing the bending energy at a point $(x, y)$ :

$$
\iint_{R^{2}}\left(\left(\frac{\partial^{2} z}{\partial x^{2}}\right)^{2}+2\left(\frac{\partial^{2} z}{\partial x \partial y}\right)^{2}+\left(\frac{\partial^{2} z}{\partial y^{2}}\right)^{2}\right) d x d y
$$

where $z$ can be defined in the $(x, y)$ plane as an in-plane interpolant or orthogonal to the $(x, y)$ plane. 
The following derivation is based on the work of Bookstein [24]. Let $G_{1}=\left(u_{1}, v_{1}\right), G_{2}=\left(u_{2}, v_{2}\right), \cdots, G_{n}=$ $\left(u_{n}, v_{n}\right)$ be the control points in sensor 1 and $G_{1}^{\prime}=$ $\left(u_{1}^{\prime}, v_{1}^{\prime}\right), G_{2}^{\prime}=\left(u_{2}^{\prime}, v_{2}^{\prime}\right), \cdots, G_{n}^{\prime}=\left(u_{n}^{\prime}, v_{n}^{\prime}\right)$ be the corresponding control points in sensor 2 . The basis function is given by $U\left(r_{i j}\right)=r_{i j}^{2} \log \left(r_{i j}^{2}\right)$, where $r_{i j}=\left|G_{i}-G_{j}\right|$, and $U(r)$ satisfies the equation for minimizing the bending energy at a point.

Then, matrices $K$ and $P$ can be defined as

$$
\begin{aligned}
\mathbf{K} & =\left[\begin{array}{cccc}
0 & U\left(r_{12}\right) & \cdots & U\left(r_{1 n}\right) \\
U\left(r_{21}\right) & 0 & \cdots & U\left(r_{2 n}\right) \\
\cdots & \cdots & \cdots & \cdots \\
U\left(r_{n 1}\right) & U\left(r_{n 2}\right) & \cdots & 0
\end{array}\right]_{n \times n}, \\
\mathbf{P} & =\left[\begin{array}{ccc}
1 & u_{1} & v_{1} \\
1 & u_{2} & v_{2} \\
\cdots & \cdots & \cdots \\
1 & u_{n} & v_{n}
\end{array}\right]_{n \times 3} .
\end{aligned}
$$

Let ${ }^{T}$ be the matrix transpose operator and $O$ be a $3 \times 3$ matrix of zeros. Then, $L$ is given as

$$
\mathbf{L}=\left[\begin{array}{c|c}
K & P \\
\hline P^{T} & O
\end{array}\right]_{(n+3) \times(n+3)} .
$$

Define $Y$ as

$$
\mathbf{Y}=\left[\begin{array}{cccc|ccc}
u_{1}^{\prime} & u_{2}^{\prime} & \cdots & u_{n}^{\prime} & 0 & 0 & 0 \\
v_{1}^{\prime} & v_{2}^{\prime} & \cdots & v_{n}^{\prime} & 0 & 0 & 0
\end{array}\right]_{2 \times(n+3)} .
$$

The affine and nonlinear deformation parameters can be estimated as

$$
L^{-1} Y^{T}=(W \mid h)^{T},
$$

where

$$
\begin{aligned}
\mathbf{W} & =\left[\begin{array}{llll}
w_{1 u} & w_{2 u} & \cdots & w_{n u} \\
w_{1 v} & w_{2 v} & \cdots & w_{n v}
\end{array}\right]_{2 \times n}, \\
h & =\left[\begin{array}{lll}
h_{1 u} & h_{2 u} & h_{3 u} \\
h_{1 v} & h_{2 v} & h_{3 v}
\end{array}\right]_{2 \times 3} .
\end{aligned}
$$

The $h_{1} \mathrm{~s}, h_{2} \mathrm{~s}$, and $h_{3} \mathrm{~s}$ are the parameters of the affine transformation, whereas the $w_{i} \mathrm{~s}$ represent the weights for the nonlinear transformation.

If a list of corresponding points between the $k$ th representative image pair is defined, the TPS function $F_{k}$ calculates the transformed coordinates $\left(x^{\prime}, y^{\prime}\right)$ as a function of the original coordinates $(x, y)$. Here, the $(x, y)$ points are in sensor 1 , and the $\left(x^{\prime}, y^{\prime}\right)$ points are the corresponding transformed points in sensor 1 . The function $F_{k}$ is defined for each pixel $(x, y)$ on sensor 1 and can be written as

$$
\begin{aligned}
\left(x^{\prime}, y^{\prime}\right)=F_{k}(x, y)= & {\left[\begin{array}{l}
h_{1 u} \\
h_{1 v}
\end{array}\right]+\left[\begin{array}{l}
h_{2 u} \\
h_{2 v}
\end{array}\right] x+\left[\begin{array}{l}
h_{3 u} \\
h_{3 v}
\end{array}\right] y } \\
& +\sum_{i=1}^{n}\left[\begin{array}{l}
w_{i u} \\
w_{i v}
\end{array}\right] U\left(\left|G_{i}-(x, y)\right|\right),
\end{aligned}
$$

where $G_{i}, i=1 . . n$, are the control points corresponding to sensor 1 . The affine parameters and the nonlinear weights can be derived in a closed-form solution according to the bending energy constraint that minimizes the curvature at every point in the grid.

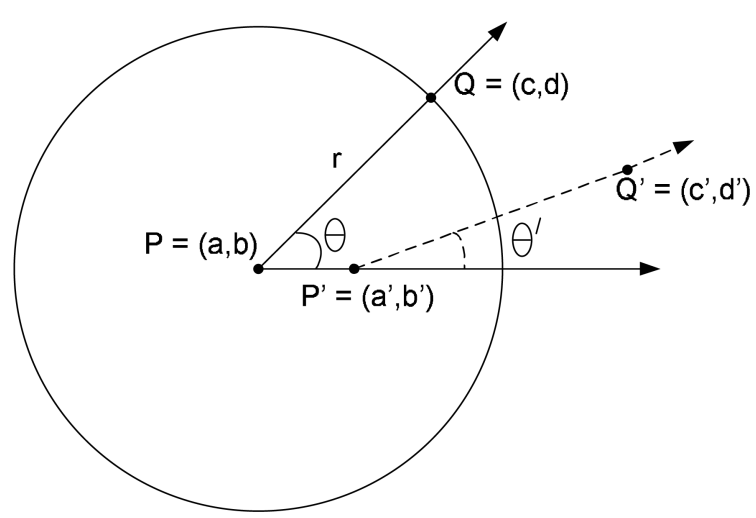

Fig. 8. Minutiae orientation is modified on the basis of the transformed minutiae location (after average deformation). $\mathrm{P}$ is the original minutiae point location, whereas $\mathrm{Q}$ is the corresponding "angle point." $\mathrm{P}^{\prime}$ and $\mathrm{Q}^{\prime}$ are the corresponding locations of $\mathrm{P}$ and $\mathrm{Q}$ after average deformation.

\subsection{Determining the Average Pixel Deformation}

If there are $m$ representative image pairs, then the application of the procedure described above will result in the generation of $m$ TPS functions, $F_{1}, F_{2}, \ldots F_{m}$. The average deformation of an arbitrary pixel $(x, y)$ on sensor 1 , denoted by $\bar{F}(x, y)$, is computed from these $m$ functions as

$$
\bar{F}(x, y)=\sum_{k=1}^{m} \frac{F_{k}(x, y)}{m} .
$$

The function $\bar{F}(x, y)$ defines the new location for each point $(x, y)$ in sensor 1 .

When (3) is used to modify the location of a minutia point (in the case of minutiae calibration), then the orientation of each minutia has to be modified also. This is accomplished by the procedure described below (also see Fig. 8).

Let $P(a, b)$ be the location of a minutia and $\theta$ be its orientation. The "angle point" $Q(c, d)$ can then be computed at a distance $r$ from $P$ as

$$
c=a+r \cos \theta, \quad d=b+r \sin \theta .
$$

Upon applying the average deformation model, the points $P(a, b)$ and $Q(c, d)$ are altered as $P^{\prime}\left(a^{\prime}, b^{\prime}\right)$ and $Q^{\prime}\left(c^{\prime}, d^{\prime}\right)$, respectively. The new orientation $\theta^{\prime}$ can now be computed as

$$
\tan \theta^{\prime}=\frac{d^{\prime}-b^{\prime}}{c^{\prime}-a^{\prime}} .
$$

\subsection{Derivation of Scaling Parameters}

The linear terms obtained from TPS are collected in a matrix:

$$
\mathbf{H}=\left[\begin{array}{ll}
h_{2 u} & h_{3 u} \\
h_{2 v} & h_{3 v}
\end{array}\right] .
$$

The $h_{1 u}$ and $h_{1 v}$ terms can be ignored as they are the translation parameters. The singular decomposition of $H$ results in $H=O_{1} D O_{2}$, where $O_{1}$ and $O_{2}$ are the rotation parameters, and $D$ is a diagonal matrix of singular values that correspond to the scaling parameters in the horizontal $S_{u}$ and vertical $S_{v}$ directions. The optimal scaling parameters $S_{U}$ and $S_{V}$ are obtained by averaging the scaling 
parameters across $m$ different pairs of representative images:

$$
S_{U}=\sum_{k=1}^{m} \frac{S_{u_{k}}}{m}, \quad S_{V}=\sum_{k=1}^{m} \frac{S_{v_{k}}}{m} .
$$

\section{EXPERIMENTAL RESULTS}

As stated earlier, the average deformation model derived in the previous section may be used for minutiae calibration or image calibration. In the case of minutiae calibration, the location and orientation of each minutia point in the fingerprint template corresponding to sensor 1 is recomputed based on the average deformation model so that it is commensurate with the minutiae template resulting from sensor 2. In image calibration, on the other hand, the location of each pixel in the original fingerprint image obtained using sensor 1 is perturbed according to the deformation function indicated in (3). Minutiae points are then extracted from this calibrated image and later utilized when matching it with images from sensor 2 .

In order to test the efficacy of the proposed calibration model, two distinct data sets were considered. The composition of these data sets and the performance of the proposed model on these data sets are reported below.

\subsection{MSU Data Set}

The MSU data set comprises of fingerprint images obtained using two different sensor technologies: an optical Digital Biometrics (DBI) sensor and a solid-state capacitive VERIDICOM (VERI) sensor [35]. The 500-dpi DBI sensor has a platen area of $1^{\prime \prime} \times 1^{\prime \prime}$ and outputs images of size $480 \times 508$. The 500-dpi VERI sensor has a sensing area of $0.6^{\prime \prime} \times 0.6^{\prime \prime}$ and outputs images of size 300 $\times$ 300. Sample images from each sensor can be seen in Fig. 5. We observe that the two images, although obtained using sensors of similar resolution, have very different spatial and photometric characteristics.

The fingerprint data of 128 different nonhabituated cooperative subjects were made available. ${ }^{3}$ All subjects provided four impressions each of four distinct fingers using both the sensors. Thus, 2,048 fingerprint impressions (four impressions each of 512 different fingers) corresponding to each sensor were available. Two different minutiaebased matchers-the BOZORTH3 matcher developed by $\mathrm{NIST}^{4}$ and the VeriFinger matcher developed by Neurotechnologija $\mathrm{a}^{5}$-were used in our experiments.

Precalibration minutiae matching results using the VeriFinger matcher are shown in Fig. 9. The matching minutiae points, as estimated by this matcher, are highlighted in this figure. Fig. 10 illustrates the difference in the minutiae count between the partial prints from VERI and the more elaborate prints from DBI. It can be observed that the DBI images contain more minutiae compared to the VERI images. This can be further confirmed in Figs. 9c and 9f, where the number of overlapping minutiae is observed to be relatively more in the DBI image pair. Further, we observe that during intersensor matching (Fig. 9i), the

3. Although data from a greater number of users were collected, only 128 of these users had data pertaining to both the sensors. For the remaining users, the corresponding images could not be determined.

4. http://fingerprint.nist.gov/NFIS/.

5. http://www.neurotechnologija.com/.
VeriFinger matcher is unsuccessful in correctly detecting corresponding minutiae pairs.

To facilitate interoperability, the calibration model was computed using eight different representative image pairs obtained from the two sensors. In our experiments, the VERI sensor was calibrated with respect to the DBI sensor. An illustration of the calibration process using mesh-grid plots can be seen in Fig. 11. Here, each mesh-grid represents the spatial geometry of individual images.

Five different matching experiments were conducted using each of the two matchers in order to demonstrate the benefit of the calibration model:

1. DBI versus DBI. The genuine and impostor match scores were generated by comparing minutiae sets within the DBI database.

2. VERI versus VERI. The genuine and impostor match scores were generated by comparing minutiae sets within the VERI database.

3. VERI versus DBI (before calibration). The genuine and impostor match scores were generated by comparing the VERI minutiae sets with the DBI minutiae sets.

4. VERI versus DBI (after minutiae calibration). The minutiae sets extracted from the VERI images were subjected to the average deformation computed using our calibration model before matching them against the minutiae sets of the DBI images. An illustration of the original minutiae set of a VERI image along with the calibrated minutiae is provided in Figs. 12a and 12b. In the current formulation, both the location and the orientation of individual minutia points are perturbed.

5. VERI versus DBI (after image calibration). The calibration model suggested here was also used to calibrate the images acquired using the VERI scanner before extracting the minutiae from them. However, the application of a nonlinear transformation to individual image pixels will generate subpixel information that can confound the minutiae detection process. In some cases, there could be a substantial perturbation of ridge information, leading to erroneous minutiae detection. One way to address this issue would be to subject the image to a simple affine transformation (predominantly scaling along the $x$ - and $y$-directions) before extracting minutiae points. In this work, these scaling factors were calculated as the average of the corresponding affine parameters generated by the calibration model based on the eight representative image pairs (refer to Section 4.3). The mesh-grid plot, along with the minutiae obtained after image calibration, is shown in Fig. 12c. As the minutiae are extracted from the "deformed" images obtained after calibration, the minutiae count might be altered.

A total of 3,072 $\left(512 *\left(\begin{array}{l}4 \\ 2\end{array}\right)\right)$ genuine scores and 523,264 impostor scores were generated for each of the five matching scenarios.

The Receiver Operating Characteristic (ROC) curves summarizing the performance of these five experiments using both the matchers are shown in Fig. 13. It is observed that the proposed calibration model results in improved intersensor matching performance. For example, in the matching scenario involving VERI versus DBI (minutiae calibration), the Genuine Accept Rate (GAR) is observed to 


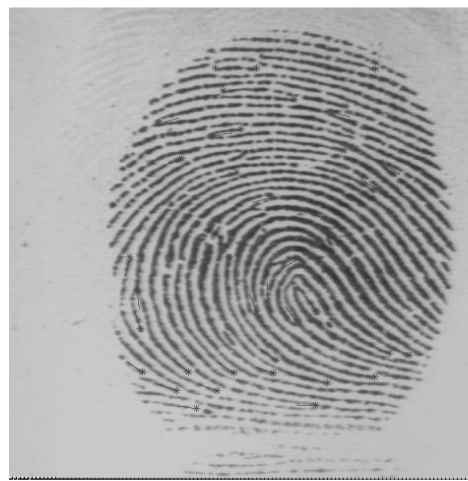

(a)

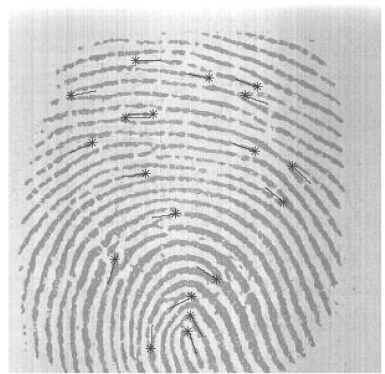

(d)

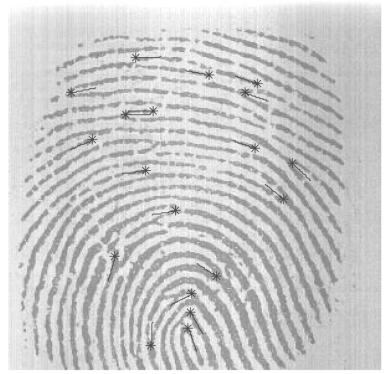

(g)

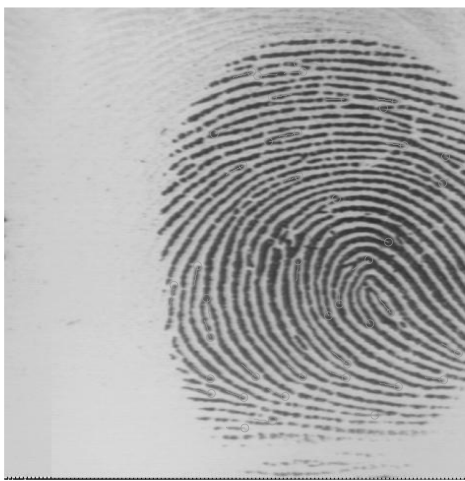

(b)

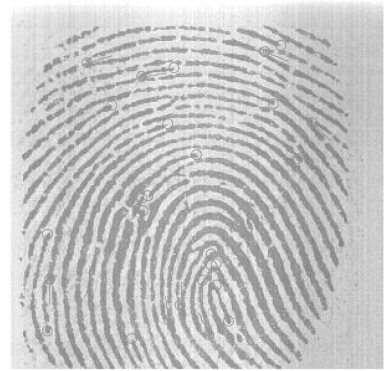

(e)

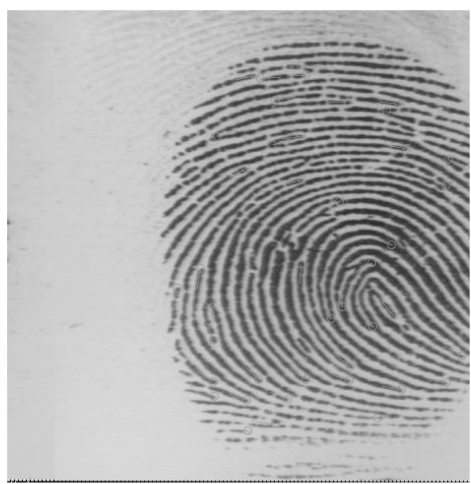

(h)

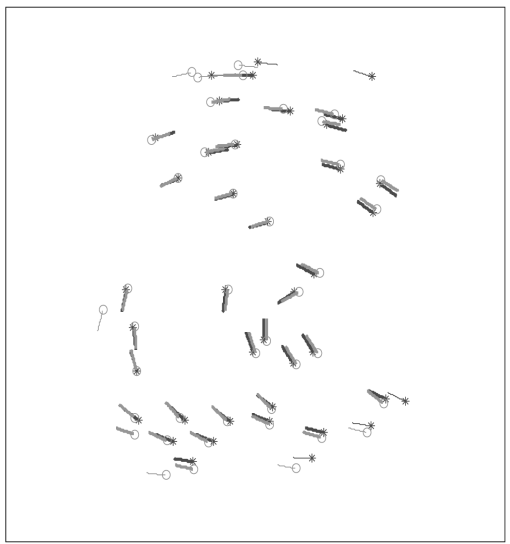

(c)

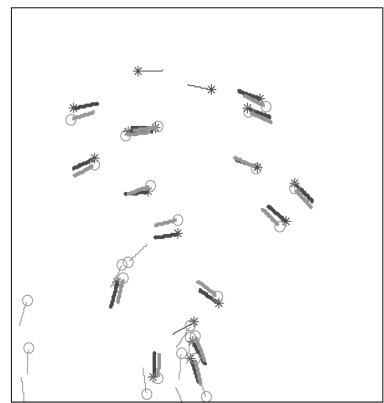

(f)

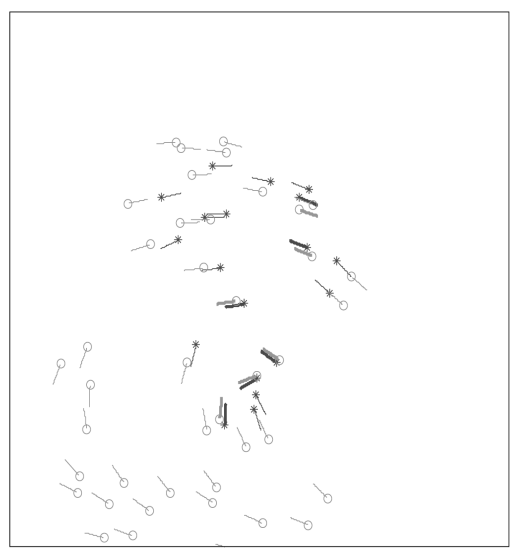

(i)

Fig. 9. Fingerprints in the first two columns are compared and the corresponding minutiae matching results are shown in the third column. The highlighted minutiae represent the minutiae that have been matched (as estimated by the VeriFinger matcher). The first row illustrates DBI versus DBI matching, the second, VERI versus VERI matching, and the third, VERI versus DBI matching. (a) DBI Fingerprint 1 . (b) DBI Fingerprint 2. (c) DBI versus DBI matching. (d) VERI Fingerprint 1. (e) VERI Fingerprint 2. (f) VERI versus VERI matching (g) VERI Fingerprint 1. (h) DBI Fingerprint 2. (i) VERI versus DBI matching.

increase from $\sim 35$ percent to $\sim 75$ percent at a False Accept Rate (FAR) of 0.01 percent when the VeriFinger matcher is used. Similarly, when the BOZORTH3 matcher is used, GAR increases from $\sim 35$ percent to $\sim 70$ percent at the same FAR. Similar observations can be made in the case of image calibration. Fig. 14 indicates the improved minutiae matching as a result of calibration.

\subsection{WVU Data Set}

The proposed model was also tested on the WVU data set ${ }^{6}$ comprising of images collected from the Crossmatch

6. The WVU data set is currently being collected at West Virginia University and is not publicly available as yet.
Verifier 300 (CM), Secugen Hamster III (SG), and Precise 100 AX fingerprint sensors (PC). CM is an optical sensor operating on the FTIR principle discussed earlier. SG is also an optical sensor but operates on the SEIR principle. PC is a capacitive sensor. The CM 500-dpi sensor has a platen area of $1.2^{\prime \prime} \times 1.2^{\prime \prime}$ and outputs images of size $480 \times 640$. The SG 500 -dpi sensor has a platen area of $0.5^{\prime \prime} \times 0.6^{\prime \prime}$ and outputs images of size $260 \times 300$. The PC capacitive sensor with an in-built Silicon sensing material from AuthenTec Inc. (AES4000) captures images at a resolution of $250 \mathrm{dpi}$ and outputs images of size $200 \times 200$.

Data were collected from 71 different nonhabituated cooperative subjects over multiple sessions. In each session, three impressions from two distinct fingers were collected 


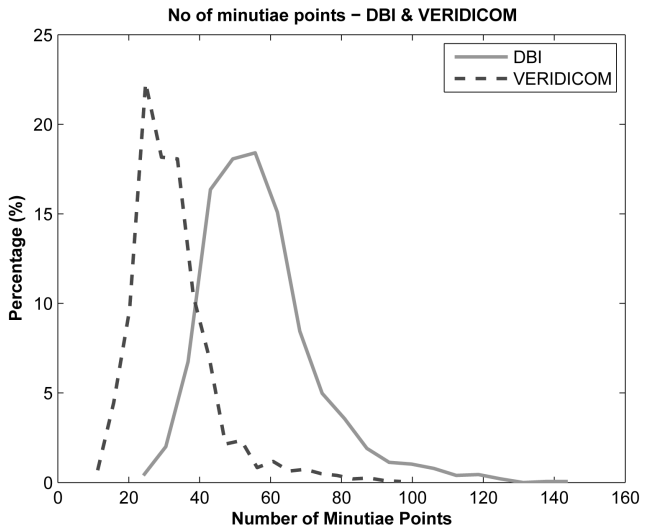

Fig. 10. The histogram of minutiae points extracted from the $\mathrm{DBI}$ and VERI images.

using all three sensors. Thus, 426 fingerprint impressions from 142 distinct fingers were collected using each sensor during a single session (Fig. 15). Some users provided data over multiple sessions, resulting in a total of 622 fingerprint impressions for each sensor.

The aforementioned data permitted us to generate 1,898 genuine scores and 45,074 impostor scores for each of the following matching experiments:

1. $\mathrm{CM}$ versus $\mathrm{CM}$,

2. SG versus $S G$,

3. PC versus PC,

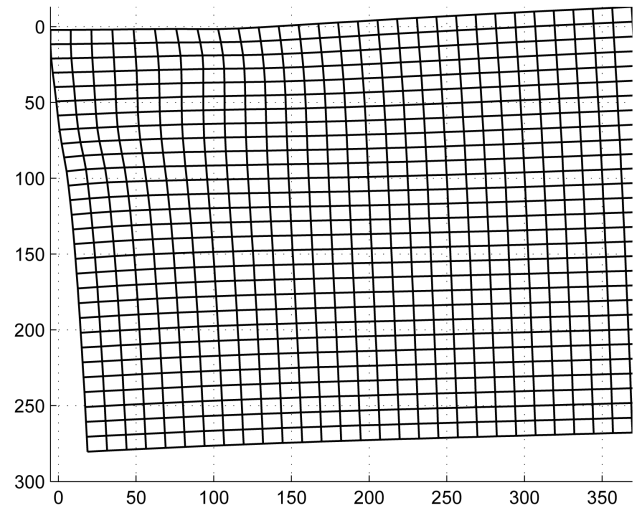

(a)

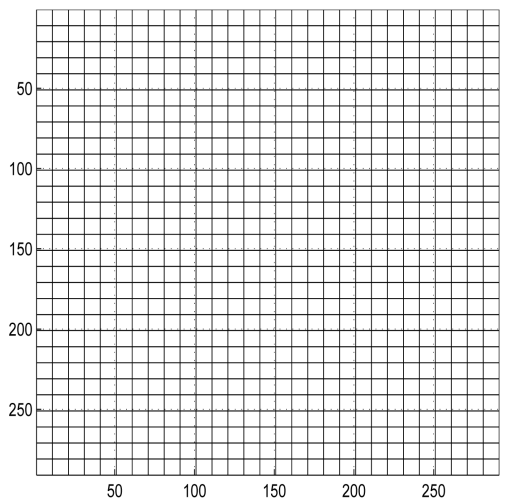

(c)
4. CM versus SG, and

5. SG versus PC.

Fig. 17 summarizes the intersensor performance on the WVU data set using the VeriFinger matcher. The following observations can be made upon viewing these ROC curves:

1. The proposed calibration model improves the intersensor matching accuracy when CM images are matched against SG images. Although both the CM and SG sensors are optical based, their operating principles are different. The calibration scheme is able to handle the geometric transformation between the two sets of images.

2. The proposed calibration model does not improve the intersensor matching accuracy when the SG images (500 dpi) are matched against the PC images (250 dpi). We conjecture that the reason for the lack of improvement is related to the fewer number of minutiae points available in the images from the PC sensor. This is substantiated by the histogram of minutiae points shown in Fig. 16. Thus, when there is significant disparity in sensor resolution and, subsequently, a large difference in image dimensions, the geometric correction algorithm proposed here cannot address the interoperability problem. This is not entirely surprising since the number of matching minutiae between two sets of points, whose cardinality is very different, will be less.

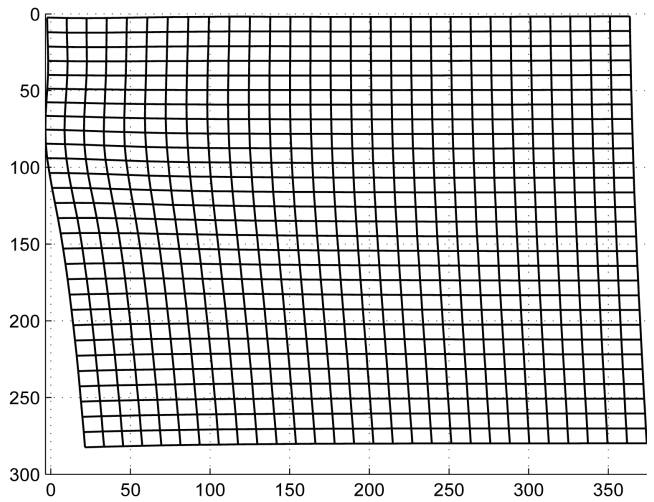

(b)

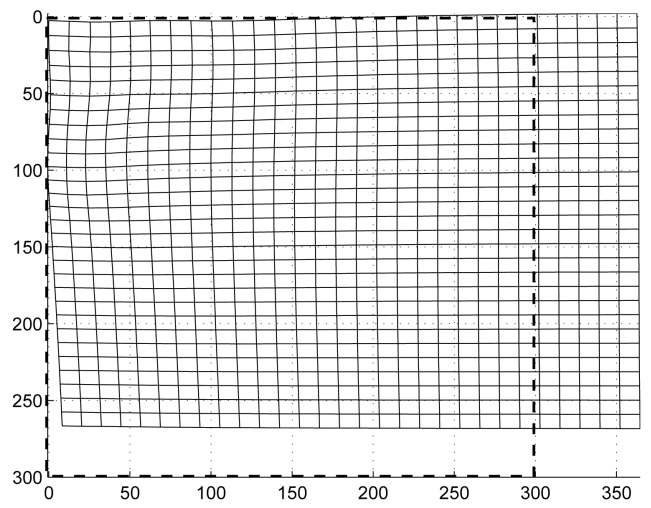

(d)

Fig. 11. Demonstration of the calibration process using mesh-grid plots. (a) and (b) The deformation of two VERI images with respect to their corresponding DBI images as estimated by the TPS model. (c) A precalibrated VERI image. (d) The postcalibrated VERI image based on the deformation of eight representative image pairs. The dashed rectangle in (d) indicates the dimensions of the original VERI image. 


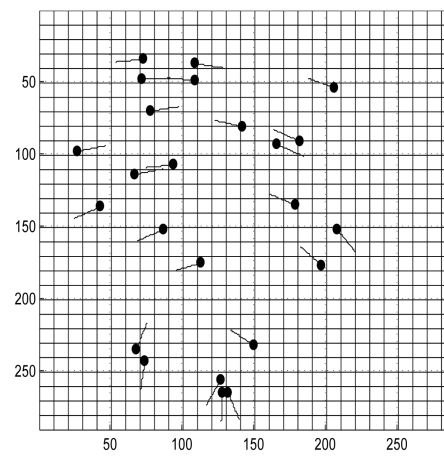

(a)

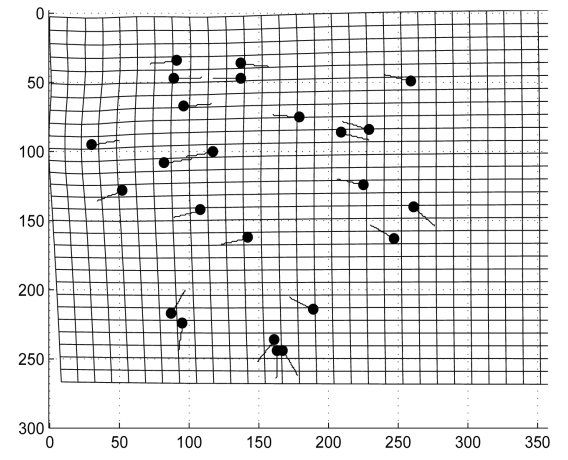

(b)

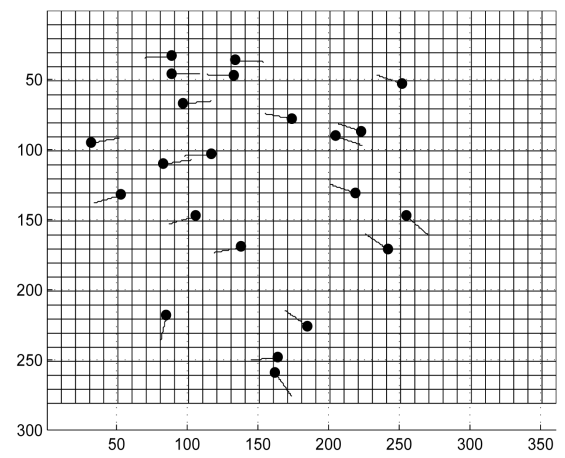

(c)

Fig. 12. (a) Original mesh-grid plot with minutiae. (b) Mesh-grid plot with calibrated minutiae. (c) Mesh-grid plot with minutiae extracted after image calibration.

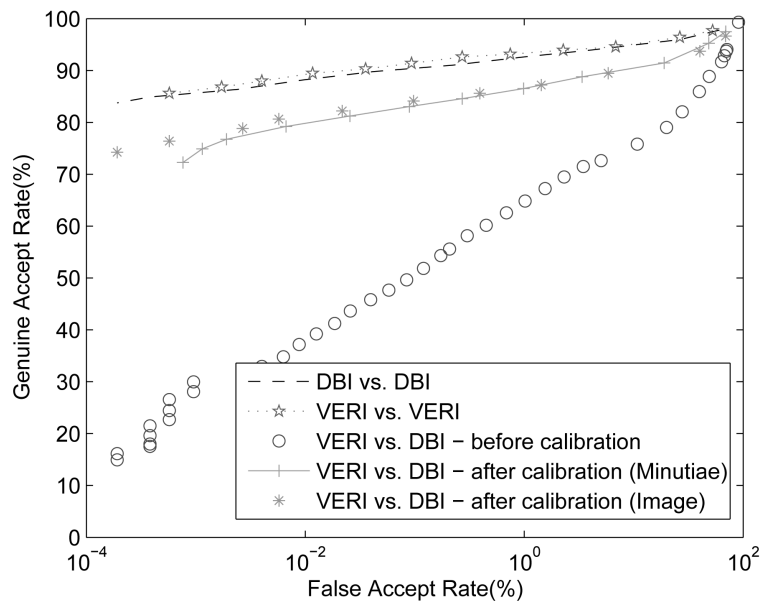

(a)

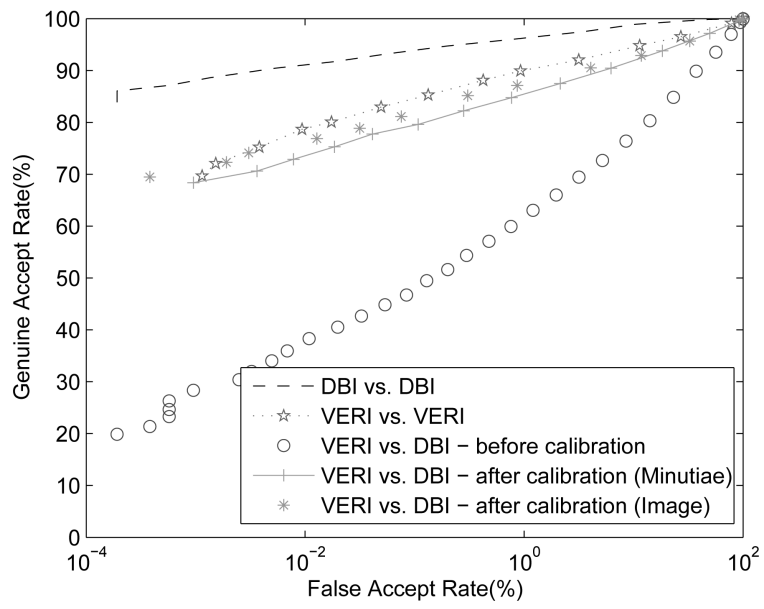

(b)

Fig. 13. ROC curves indicating the improvement in intersensor performance on the MSU database. (a) VeriFinger matcher. (b) BOZORTH3 matcher.

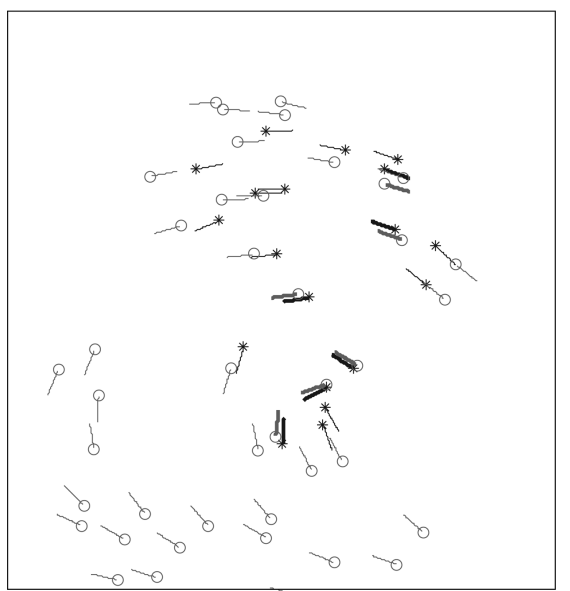

(a)

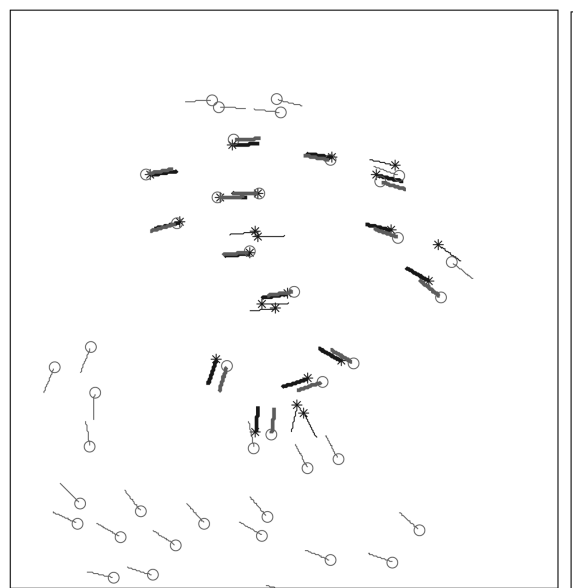

(b)

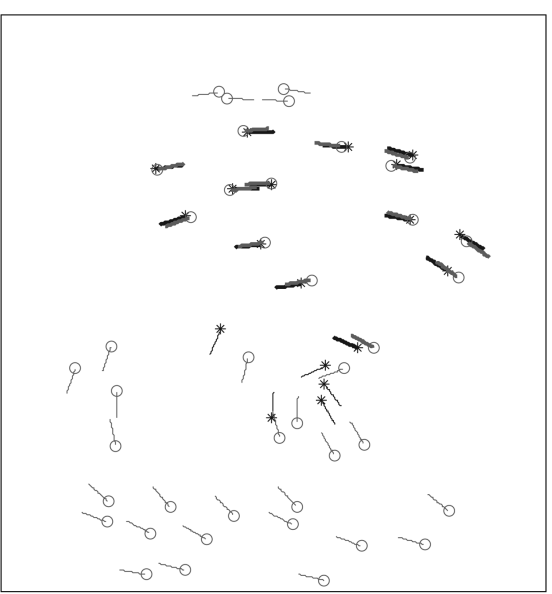

(c)

Fig. 14. Improved intersensor minutiae matching after calibration. In (a), the matching minutiae before calibration is indicated. In (b) and (c), the matching minutiae after image and minutiae calibration, respectively, are shown. Matching minutiae pairs are highlighted to distinguish them from the other minutiae points. Match scores as assessed by the VeriFinger matcher for (a), (b) and (c) are 35, 193, and 202, respectively. (a) VERI versus $\mathrm{DBI}$-before calibration. (b) VERI versus DBI-after image calibration. (c) VERI versus DBI-after minutiae calibration.

\subsection{Computational Complexity}

The incorporation of the calibration model in a generic fingerprint system to facilitate interoperability requires two primary steps: 1) estimating the calibration parameters using a small subset of image pairs $(\sim 8-10)$ and 2$)$ perturbing the minutiae set (or images) associated with one of the sensors using the calibration parameters. Step 1 has to be executed only once for a pair of sensors. Step 2 has to be executed for 


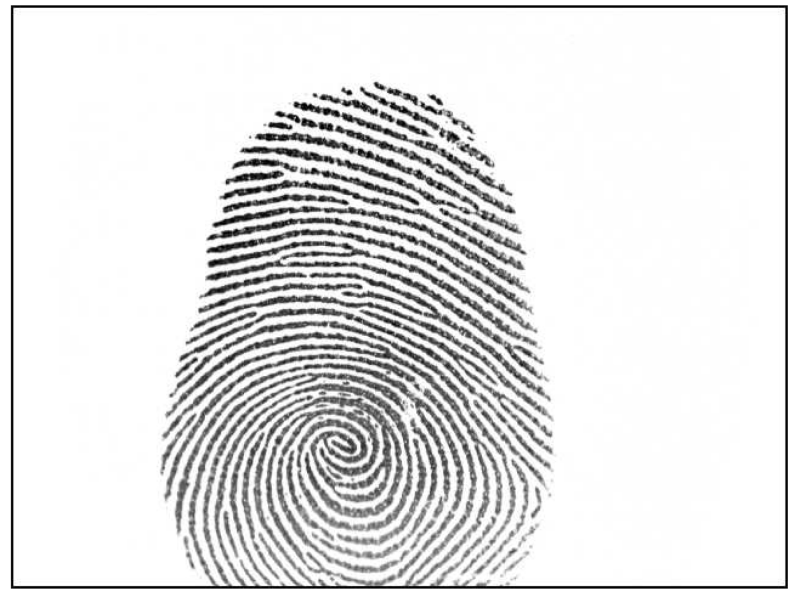

(a)

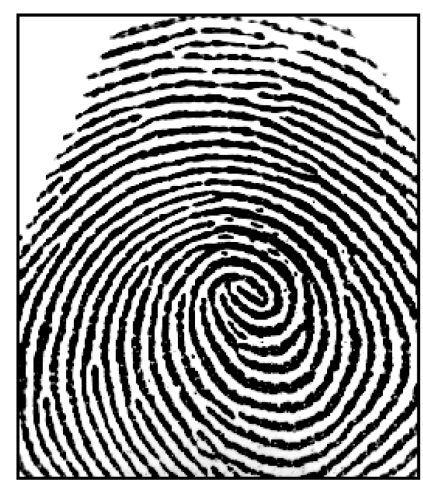

(b)

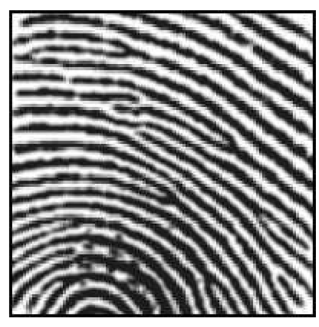

(c)

Fig. 15. Sample images from the WVU data set. Images of the same finger were acquired using three different sensors. (a) CM. (b) SG. (c) PC.

each minutiae set (or image) associated with one of the sensors. Below, we analyze the complexity of these two steps.

Let $n$ denote the number of minutiae extracted from a single fingerprint image. The computational complexity of step 1 depends on the complexity of estimating the TPS parameters and evaluating the TPS function (Section 4). The complexity of the former is $O\left(n^{3}\right)$, and that of the latter is $O\left(n^{2}\right)$. These operations can be accelerated as shown in [36] and [37]. Estimating the TPS parameters and the average deformation model is an offline process that is conducted prior to fingerprint matching and does not affect the speed of the matching algorithm employed in real time.

During step 2, the average deformation model is applied to each minutiae point (or to each pixel) present in the minutiae set of one of the sensors. On a computer equipped with a dual processor (Intel Xeon CPUs of $2.4 \mathrm{GHz}$ and $2.39 \mathrm{GHz}$ ) and 512 Mbytes of RAM, the time required to compute the transformed coordinates $\left(x^{\prime}, y^{\prime}\right)$ as a function of the original coordinates $(x, y)$ is about 6.6 sec. $^{7}$

\section{Discussion AND FUtURE WORK}

In this paper, we have demonstrated that a simple nonlinear calibration scheme based on the TPS model is useful to handle variations in minutiae distributions across multiple sensors. The parameters of the model (average deformation) are computed based on a small representative set of image pairs containing control points (landmarks) whose correspondences are manually established. The average deformation model is used to distort the minutiae points of images acquired using one sensor before comparing them with the minutiae points of images corresponding to another sensor. A significant performance improvement is observed when the proposed scheme is utilized to compare fingerprint images originating from two different sensors. Only a few representative image pairs are needed for the successful implementation of the proposed method. In the future, we plan to use more sophisticated calibration grids by imaging rigid fingerlike synthetic material with preestablished control points. This would avoid issues related to the user-dependent elasticity of the skin.

7. This is for a minutiae set containing 28 points.
An inherent limitation of the proposed scheme is the inability to compensate for variations introduced in fingerprint images acquired using sensors with significant differences in resolution and local geometric distortions. A simple nonlinear transformation may not be sufficient to register such disparate images. Also, the proposed approach is not designed to handle photometric variations across sensors. This important consideration will be a topic for future research.

The proposed model assumes that the intersensor distortion can be approximated using TPSs. It may be instructive to evaluate alternate models based on nonparametric local perturbations (such as optical flow) to describe these deformations. We also plan to extend our approach toward addressing compatibility between slap and rolled prints.

\section{ACKNOWLEDGMENTS}

The authors would like to thank Dr. Anil Jain from Michigan State University (MSU) for granting them access to the MSU Fingerprint Database. This project was funded in part by the

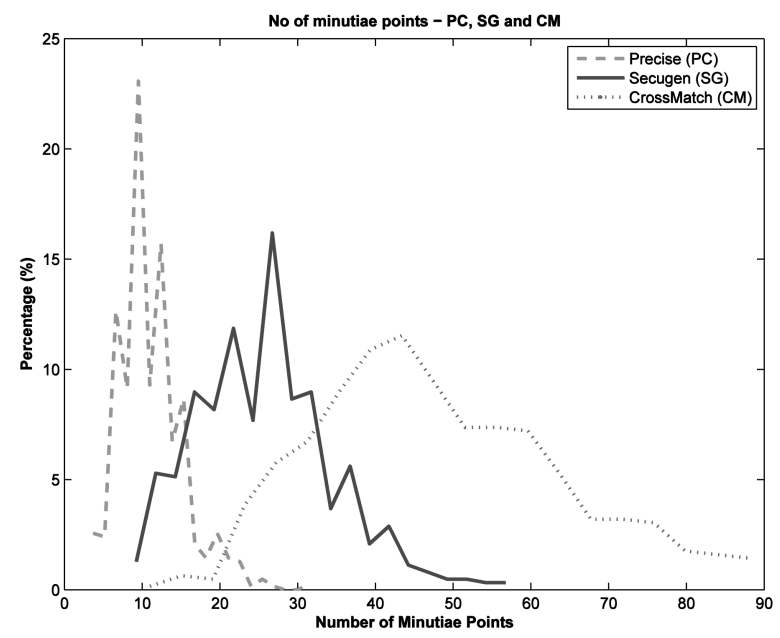

Fig. 16. The histogram of minutiae points extracted from the PC, SG, and $\mathrm{CM}$ images. 


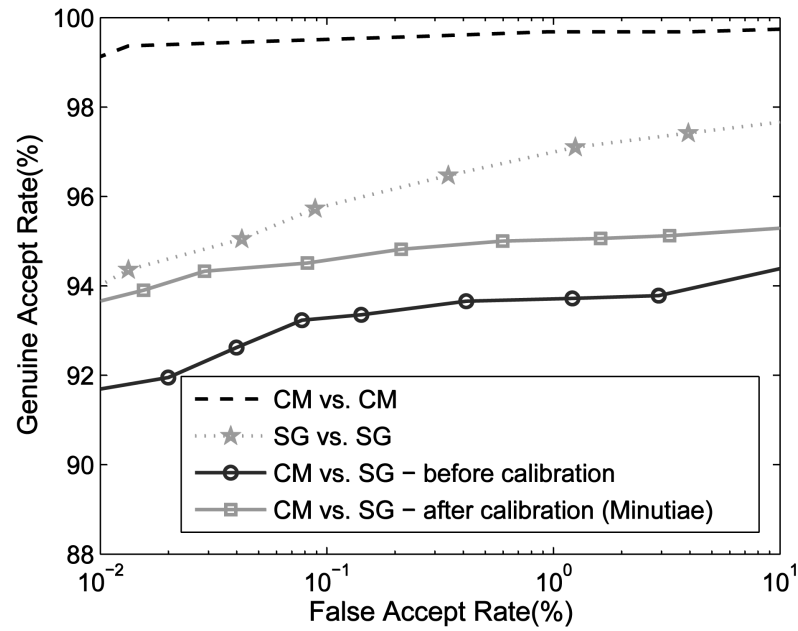

(a)

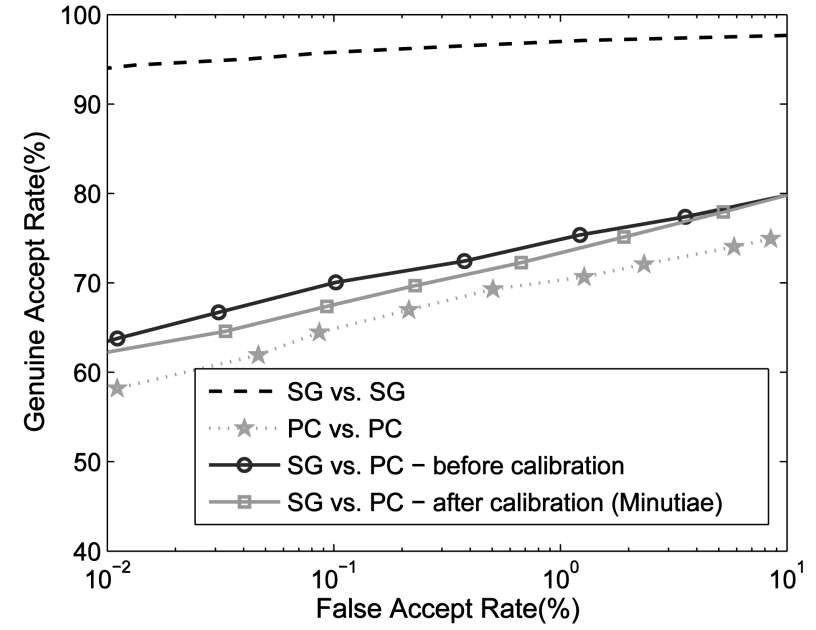

(b)

Fig. 17. ROC curves indicating the intersensor performance on the WVU database using the VeriFinger matcher. (a) CM versus SG. (b) SG versus PC.

Center for Identification Technology Research (CITeR), West Virginia University. This work was done when Rohan Nadgir was a graduate student at West Virginia University. Arun Ross was supported by US National Science Foundation CAREER grant number IIS 0642554.

\section{REFERENCES}

[1] D. Maltoni, D. Maio, A.K. Jain, and S. Prabhakar, Handbook of Fingerprint Recognition. Springer, July 2003.

[2] S. Pankanti, S. Prabhakar, and A.K. Jain, "On the Individuality of Fingerprints," IEEE Trans. Pattern Analysis and Machine Intelligence, vol. 24, no. 8, pp. 1010-1025, Aug. 2002.

[3] A. Hicklin and C. Reedy, "Implications of IDENT/IAFIS Image Quality Study for Visa Fingerprint Processing," technical report, Mitretek Systems, Oct. 2002.

[4] A. Ross and A. Jain, "Biometric Sensor Interoperability: A Case Study in Fingerprints," Proc. Int'l European Conf. Computer Vision Workshop Biometric Authentication (BioAW '04), pp. 134-145, May 2004.

[5] A. Martin, M. Przybocki, G. Doddington, and D. Reynolds, "The NIST Speaker Recognition Evaluation-Overview, Methodology, Systems, Results, Perspectives (1998)," Speech Comm., vol. 31, pp. 225-254, 2000.

[6] Int'l Biometric Group, “Independent Testing of Iris Recognition Technology (ITIRT)-Final Report," http://www.biometric group.com/reports/public/reports/ITIRT_report.htm, May 2005.

[7] P.J. Phillips, A. Martin, and C.1. Wilson, M. Przybocki, "An Introduction to Evaluating Biometric Systems," Computer, vol. 33, no. 2, pp. 56-63, Feb. 2000.

[8] T. Faltemier and K. Bowyer, "Cross Sensor 3D Face Recognition Performance," Proc. SPIE Conf. Biometric Technology for Human Identification III, Apr. 2006.

[9] F. Podio, J. Dunn, L. Reinert, C. Tilton, L. O'Gorman, P. Collier, M. Jerde, and B. Wirtz, Common Biometric Exchange File Format (CBEFF), NIST Interagency Report 6529, NIST, 1999.

[10] R.M. Bolle, N.K. Ratha, A. Senior, and S. Pankanti, "Minutia Template Exchange Format," Proc. IEEE Workshop Automatic Identification Advanced Technologies, pp. 74-77, 1999.

[11] CJIS, "Electronic Fingerprint Transmission Specification-Appendix F," Technical Report CJIS-RS-0010 (V7), Criminal Justice Information Services Division, Dept. of Justice-Fed. Bureau of Investigation, Jan. 1999.

[12] B. Ulery, A. Hicklin, C. Watson, M. Indovina, and K. Kwong, "Slap Fingerprint Segmentation Evaluation 2004," NIST Interagency Report 7209, NIST and Mitretek Systems, Mar. 2005.

[13] Secugen Biometric Solutions, "SEIR Optic Technology," white paper, Secugen, http://www.secugen.com/company/ip.htm, 2004
[14] M.S. Ennis, R.K. Rowe, S.P. Corcoran, and K.A. Nixon, "Multispectral Sensing for High-Performance Fingerprint Biometric Imaging," white paper, Lumidigm, Inc., http://www.lumidigm. com/lightPrint.html, 2007.

[15] X. Xia and L. O'Gorman, "Innovations in Fingerprint Capture Devices," Pattern Recognition, vol. 36, no. 2, pp. 361-369, Feb. 2003.

[16] H.H. Teng and S.C. Jo, Method and Apparatus for Reduction of Trapezoidal Distortion and Improvement of Image Sharpness in an Optical Image Capturing System, US Patent 6,324,020 B1, Nov. 2001.

[17] H.H. Teng and S.C. Jo, High Contrast Low Distortion Optical Acquisition System for Image Capturing, US Patent 6,381,347 B1, Apr. 2002.

[18] A. Senior and R. Bolle, "Improved Fingerprint Matching by Distortion Removal," IEICE Trans. Information and Systems, vol. E84-D, pp. 825-831, 2001.

[19] Z. Zhang, "Flexible Camera Calibration by Viewing a Plane from Unknown Orientations," Proc. Seventh Int'l Conf. Computer Vision (ICCV'99), pp. 666-673, 1999.

[20] J.-M. Lavest, M. Viala, and M. Dhome, "Do We Really Need an Accurate Calibration Pattern to Achieve a Reliable Camera Calibration," Proc. Fifth European Conf. Computer Vision (ECCV '98), vol. 1, pp. 158-174, 1998.

[21] T. Clarke and J. Fryer, "The Development of Camera Calibration Methods and Models," Photogrammetric Record, vol. 16, no. 91, pp. 51-66, Apr. 1998.

[22] J. Heikkila and O. Silven, "A Four-Step Camera Calibration Procedure with Implicit Image Correction," Proc. IEEE Conf. Computer Vision and Pattern Recognition (CVPR '97), p. 1106, 1997.

[23] A. Ross, S. Dass, and A. Jain, "A Deformable Model for Fingerprint Matching," Pattern Recognition, vol. 38, no. 1, pp. 95103, Jan. 2005.

[24] F.L. Bookstein, "Principal Warps: Thin-Plate Splines and the Decomposition of Deformations," IEEE Trans. Pattern Analysis and Machine Intelligence, vol. 11, no. 6, pp. 567-585, June 1989.

[25] K. Rohr, M. Fornefett, and H.S. Stiehl, "Approximating Thin-Plate Splines for Elastic Registration: Integration of Landmark Errors and Orientation Attributes," Proc. 16th Int'l Conf. Information Processing in Medical Imaging (IPMI '99), pp. 252-265, June/July 1999.

[26] K. Rohr, H. Stiehl, R. Sprengel, W. Beil, T. Buzug, J. Weese, and M. Kuhn, "Point-Based Elastic Registration of Medical Image Data Using Approximating Thin-Plate Splines," Lecture Notes in Computer Sscience 1131, pp. 297-306, 1996.

[27] K. Rohr, R. Sprengel, and H. Stiehl, "Incorporation of Landmark Error Ellipsoids for Image Registration Based on Approximating Thin-Plate Splines," Proc. 11th Int'l Symp. and Exhibition Computer Assisted Radiology and Surgery (CAR '97), June 1997.

[28] K. Rohr, "Image Registration Based on Thin-Plate Splines and Local Estimates of Anisotropic Landmark Localization Uncertainties," Proc. First Int'l Conf. Medical Image Computing and ComputerAssisted Intervention (MICCAI '98), pp. 1174-1183, 1998. 
[29] A.C. Evans, W. Dai, D.L. Collins, P. Neelin, and S. Marrett, "Warping of a Computerized 3-D Atlas to Match Brain Image Volumes for Quantitative Neuroanatomical and Functional Analysis," Proc. SPIE Medical Imaging V: Image Processing, M.H. Loew, ed., vol. 1445, pp. 236-246, June 1991.

[30] A.M. Bazen and S.H. Gerez, "Fingerprint Matching by Thin-Plate Spline Modelling of Elastic Deformations," Pattern Recognition, vol. 36, no. 8, pp. 1859-1867, Aug. 2003.

[31] A. Almansa and L. Cohen, "Fingerprint Image Matching by Minimization of a Thin-Plate Energy Using a Two-Step Algorithm with Auxiliary Variables," Proc. Fifth IEEE Workshop Applications of Computer Vision (WACV'00), pp. 35-40, 2000.

[32] A.M. Bazen and S.H. Gerez, "Elastic Minutiae Matching by Means of Thin-Plate Spline Models," Proc. 16th Int'l Conf. Pattern Recognition (ICPR '02), vol. 2, 2002.

[33] Y. Chen, S. Dass, A. Ross, and A. Jain, "Fingerprint Deformation Models Using Minutiae Locations and Orientations," Proc. Seventh IEEE Workshop Application of Computer Vision/IEEE Workshop Motion and Video Computing (WACV/MOTION '05), vol. 1, pp. 150-155, 2005.

[34] A. Ross, S. Shah, and J. Shah, "Image versus Feature Mosaicing: A Case Study in Fingerprints," Proc. SPIE Conf. Biometric Technology for Human Identification III, Apr. 2006.

[35] A. Jain, S. Prabhakar, and A. Ross, "Fingerprint Matching: Data Acquisition and Performance Evaluation," Technical Report MSUCPS-99-14, Michigan State Univ., Mar. 1999.

[36] G. Donato and S. Belongie, "Approximate Thin Plate Spline Mappings," Proc. Seventh European Conf. Computer Vision (ECCV '02), vol. 3, pp. 21-31, 2002.

[37] A. Zandifar, S. Lim, R. Duraiswami, N. Gumerov, and L. Davis, "Multi-Level Fast Multipole Method for Thin Plate Spline Evaluation," Proc. Int'l Conf. Image Processing (ICIP '04), vol. 3, pp. 1683-1686, 2004.

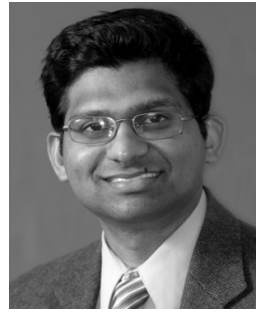

Arun Ross received the BE (Hons.) degree in computer science from the Birla Institute of Technology and Science, Pilani, India, in 1996, and the MS and $\mathrm{PhD}$ degrees in computer science and engineering from Michigan State University, East Lansing, in 1999 and 2003, respectively. Between 1996 and 1997, he was with the Design and Development Group of Tata Elxsi (India) Ltd., Bangalore, India. He also spent three summers (2000-2002) with the Imaging and Visualization Group at Siemens Corporate Research, Inc., Princeton, New Jersey, working on fingerprint recognition algorithms. He is currently an assistant professor in the Lane Department of Computer Science and Electrical Engineering, West Virginia University, Morgantown. He is actively involved in the development of the pattern recognition and biometrics curricula at West Virginia University. His research interests include pattern recognition, classifier combination, machine learning, computer vision, and biometrics. He is a coauthor of the book Handbook of Multibiometrics and a coeditor of the book Handbook of Biometrics, both published by Springer. He is a recipient of the US National Science Foundation CAREER Award and was an invited speaker at the Frontiers of Science Symposium organized by the National Academy of Sciences in November 2006. He also served as one of the US experts in multibiometrics at the ISO/IEC JTC1 SC37 meeting for biometric standards in 2004. He is a member of the IEEE.

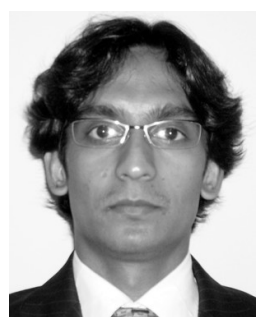

Rohan Nadgir received the BE degree in electronics and telecommunications engineering from the Maharashtra Institute of Technology, Pune, India, in 2002, and the MS degree in electrical engineering from West Virginia University, Morgantown, in 2006. He is currently working as an algorithm scientist with Bio-Key International, Minneapolis. His research interests include image processing, biometrics, pattern recognition, and computer vision.

$\triangleright$ For more information on this or any other computing topic, please visit our Digital Library at www.computer.org/publications/dlib. 